\title{
Après maxillectomie : reconstruire ou obturer ?
}

\author{
Marilena Fistes ${ }^{1}$, Marie-Alix Fauroux ${ }^{1}$, Jacques Margerit ${ }^{1}$, \\ Jacques Yachouch ${ }^{2}$, Renaud Garrel ${ }^{3}$
}

${ }^{1}$ Faculté d'Odontologie, 545 avenue Pr JL Viala, 34193, Montpellier Cedex 5, France

${ }^{2}$ Service de Chirurgie maxillo-faciale

${ }^{3}$ Service d'ORL

marilenafistes@hotmail.com

L'exérèse tumorale au maxillaire entraîne une mutilation du massif facial à l'origine d'un déficit fonctionnel et esthétique, avec une forte répercussion sociale. La réhabilitation de la perte de substance maxillaire (PDSM) peut se faire par reconstruction chirurgicale ou par prothèse maxillo-faciale (PMF). Il existe peu d'études comparatives et le choix entre ces deux techniques reste assez subjectif. Il semble difficile de s'en tenir au seul volume de la PDSM (Okay et al. 2001, Santamaria et Cordeiro 2006). La décision doit aussi tenir compte de l'âge du patient, de ses antécédents médicaux, et de l'expérience technique de l'équipe médicale.

La réhabilitation prothétique ne provoque pas de contrainte physique supplémentaire pour le patient et, selon le site anatomique, elle peut être plus esthétique que la chirurgie (Schaaf 1984) ; le coût de réalisation de la PMF est faible; sa mise en œuvre est rapide et elle offre une possibilité de réhabilitation dentaire relativement rapide en ambulatoire; enfin, la PMF autorise une bonne surveillance carcinologique (Borlase 2000). La PMF peut s'adapter à la majorité des patients et des cas cliniques; elle répond donc à toutes les contre-indications de la chirurgie reconstructrice. En revanche, aucune prothèse n'est "définitive »; l'utilisation d'un obturateur prothétique volumineux peut être difficile ; il est compliqué d'obtenir une étanchéité et une élocution parfaites (Edgerton et Zovickian 1956, Shestak et al. 1988); et bien sûr, la PDSM est toujours présente. Un enchaînement de contraintes se succède du fait de la reconstruction artificielle : problèmes fonctionnels, mécaniques, physiologiques, esthétiques, psychologiques, et aussi problèmes d'hygiène et d'entretien.

Évitant les inconvénients de l'obturateur, la chirurgie reconstructrice constitue la forme la plus parfaite de réhabilitation. Elle masque "définitivement " la perte de substance et permet une étanchéité excellente (Benoist 1982). Elle présente cependant des inconvénients (Converse 1977) : en particulier, elle nécessite un geste supplémentaire qui peut impliquer des complications au site donneur; le plus souvent, le résultat chirurgical n'est pas immédiat, et l'attente du résultat final se fait dans des conditions parfois difficiles et douloureuses ; un terrain irradié peut compliquer la chirurgie ; enfin, la surveillance carcinologique est compliquée sur un lambeau ou un tissu greffé.

La prise en charge des PDSM semble évoluer vers une alliance des techniques de la chirurgie microvasculaire, de la distraction osseuse, de l'implantologie et de la réhabilitation prothétique par CFAO (conception et fabrication assistée par ordinateur) pour surmonter les difficultés de la PMF actuelle, en particulier pour obtenir une bonne étanchéité et un équilibre prothétique approprié (Armbruster 2004). Que le choix se porte sur une reconstruction chirurgicale ou prothétique, la réhabilitation psychologique et sociale est essentielle, dès le stade de préparation de l'intervention. Dans tous les cas, la notion d'équipe pluridisciplinaire s'impose pour une prise en charge optimale des patients. 